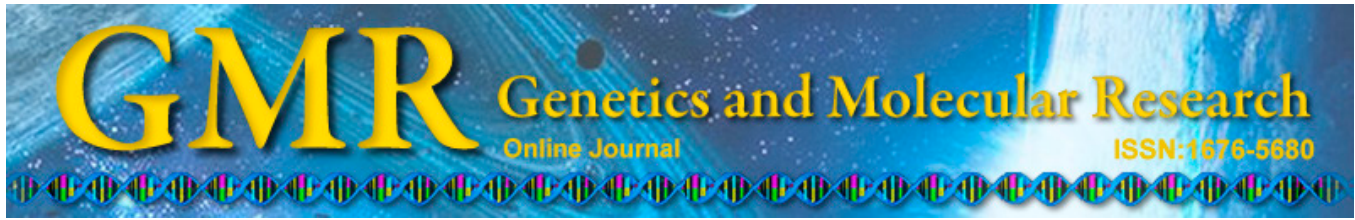

\title{
Adeno-associated virus-mediated BMP-7 and SOX9 in vitro co-transfection of human degenerative intervertebral disc cells
}

\author{
X.F. Ren*, Z.Z. Diao*, Y.M. Xi, Z.H. Qi, S. Ren, Y.J. Liu, D.L. Yang, \\ X. Zhang and S.L. Yuan \\ Department of Orthopaedics, Affiliated Hospital of Qingdao University, \\ Qingdao, China \\ *These authors contributed equally to this study. \\ Corresponding author: Y.M. Xi \\ E-mail: yongmingxidoc@126.com
}

Genet. Mol. Res. 14 (2): 3736-3744 (2015)

Received March 13, 2014

Accepted July 1, 2014

Published April 22, 2015

DOI http://dx.doi.org/10.4238/2015.April.22.1

\begin{abstract}
Bone morphogenetic protein-7 (BMP-7) and SOX9 are important transcription factors in chondrogenesis. In this study, we examined the biological function of the adeno-associated virus (AAV)mediated BMP-7 and SOX9 double gene in vitro co-transfection of nucleus pulposus cells of human degenerative intervertebral disc. Human intervertebral disc nucleus pulposus cells were cultured in vitro and subcultured for 5 generations. Using rAAV-BMP-7 and rAAV-SOX9 AAV2-type AAV viruses, the cells were divided into 4 groups: blank normal, BMP-7 transfection, SOX9 transfection, and BMP-7 and SOX9 co-transfection. After $48 \mathrm{~h}$, expression of type II collagen and its mRNA in nucleus pulposus cells was determined. The expression of type II collagen in BMP-7 transfection, SOX9 transfection, and co-transfection groups was up-regulated to varying degrees compared to the blank control group. The type II collagen mRNA level expression in the co-transfection group was significantly higher than in other groups $(\mathrm{P}<0.05)$. AAV-mediated BMP-7 and
\end{abstract}


SOX9 in vitro co-transfection can promote the synthesis of type II collagen in nucleus pulposus cells in the human degenerative intervertebral disc. Double-gene therapy has a synergistic effect in the treatment of intervertebral disc degeneration.

Key words: Adeno-associated virus; Bone morphogenetic protein-7; SOX9; Type II collagen

\section{INTRODUCTION}

Lumbar disc degeneration is an important cause of low back pain, and the delay and reversal of degenerative intervertebral disc is a key factor in research of spine surgery (Borenstein, 2000). With continuous improvement of treatment methods involving gene therapy, the reversal of the degenerative intervertebral disc has received increasing attention. Thus far, encouraging results have been achieved (Wang et al., 1999). Wehling et al. (1997) first proposed the transgenic reversal of intervertebral disc degeneration, and successfully achieved transfection of a target gene into in vitro cultured cartilage cells of cartilage endplate in a bovine intervertebral disc. Sai et al (2007) used transforming growth factor (TGF)- $\beta 1,3$ as a target gene and adenovirus as vector to transfect rabbit intervertebral disc nucleus pulposus cells, which showed increased type II collagen and proteoglycan synthesis. Wallach et al. (2003) successfully constructed recombinant adenovirus vector adenovirus-tissue inhibitor of metalloproteases (TIMPs) carrying TIMPs and in vivo transfected them into human intervertebral disc cells, which showed increased proteoglycan synthesis.

Although single-gene transfection of intervertebral disc cells has shown good results, a large quantity of viral vectors is needed, which increases the risk of cytotoxicity and the induction of an immune response (Lohr et al., 2001). Co-transfection of 2 or more factors into intervertebral disc cells has been examined to reduce the dosage of viral vectors required and to produce improved biological effects. Xi et al. (2013b) constructed adeno-associated virus (AAV)-hVEGF ${ }_{165}$ and AAV-TGF- $\beta 1$ for transfection or co-transfection, respectively, of intervertebral disc cells. The results showed that the expression quantity of type II collagen of AV-hVEGF ${ }_{165}$ and AAV-TGF- $\beta 1$ double-gene co-transfection of annulus fibrosus cells significantly increased compared with single-gene transfection. Wallach et al. (2003) combined TGF- $\beta 1$, bone morphogenetic protein (BMP)-2, and insulin-like growth factor 1 for transfection of intervertebral disc cells. The combination of these 3 factors showed clear biological effects compared to the use of 1 or 2 factors.

In this study, we used AAV-mediated BMP-7 and SOX9-double gene for in vitro cotransfection of nucleus pulposus cells of the human degenerative intervertebral disc using SOX9 to promote matrix expression function and BMP-7 to promote the synthesis of marrow stromal cell components and up-regulate the expression function of SOX9. The effect of double-gene therapy on human intervertebral disc degeneration was determined.

\section{MATERIAL AND METHODS}

\section{Culture of human degenerative intervertebral disc nucleus pulposus cells}

Intervertebral disc nucleus pulposus cells from an aborted fetus were placed in F12 cul- 
ture medium containing $20 \%$ fetal bovine serum and the cells were incubated at $37^{\circ} \mathrm{C}$ with $5 \%$ $\mathrm{CO}_{2}$ for culture. After fusion, the cells were subcultured by digestion with $0.25 \%$ trypsin. The multi-subculture method was used to produce degenerative intervertebral disc cells by dedifferentiation. The entire experimental process complied with the ethical standards of the institution.

\section{AAV transfection and determination of transfection rate}

rAAV-BMP-7 and rAAV-SOX9 were provided by Shenzhen Biowit Technologies Ltd. (Shenzhen, China). In accordance with the multiplicity of infection of $1 \times 10^{4}$, rAAV2-EGFP was mixed with serum-free F12 culture fluid; the final volume just covered the surface of the cells. This solution was added to the cultured cells washing with phosphate-buffered saline, followed by incubation at $37^{\circ} \mathrm{C}$ for $10-30 \mathrm{~min}$. Next, the serum-free F12 culture medium was used to wash the cells 3 times to remove the unadsorbed rAAV2-EGFP. F12 culture liquid containing $21 \%$ fetal bovine serum was added, followed by culturing of the cells at $37^{\circ} \mathrm{C}$ in $5 \%$ $\mathrm{CO}_{2}$. After transfection for $72 \mathrm{~h}$, EGFP expression in cells was observed under a fluorescence microscope. Cells showing green fluorescence were considered to be positive. The percentage of positive cells of 100 cells was considered to be the transfection rate.

\section{rAAV2-BMP-7 and rAAV2-SOX9 co-transfection of degenerative intervertebral disc nucleus pulposus cells}

The 5th generation of nucleus pulposus cells was inoculated onto a 12-well plate. After $24 \mathrm{~h}$, the cells were well adherent to the wall, and transfection was started. In accordance with the multiplicity of infection of $1 \times 10^{4}$, the rAAV2-SOX9, rAAV2-BMP-7 and double-gene mixture (1:1) were mixed with F12 culture liquid not containing the double antibody, respectively, and then was added to the culture well, followed by culturing at $37^{\circ} \mathrm{C}$ and under $5 \% \mathrm{CO}_{2}$. After $10 \mathrm{~h}$, the liquid was removed, and F12 culture medium containing $10 \%$ fetal bovine serum was added, followed by continued culture. The experiments were divided into 4 groups: blank control; BMP-7 transfection; SOX9 transfection; BMP-7 and SOX9 co-transfection.

\section{Determination of type II collagen mRNA}

The 5th generation of nucleus pulposus cells was inoculated onto a 12-well plate. rAAV2-BMP-7, rAAV2-SOX9 and double-gene transfection were performed as described above (experimental groups). Third-generation nucleus pulposus cells that had not been subjected to transfection were used as controls. After $48 \mathrm{~h}$, the culture medium was removed and the cells were collected. Total RNA in each group was extracted using TRIzol, and the purity and concentration of the RNA were measured. The type II collagen mRNA expression level was detected using fluorescence quantitative PCR. All primers were constructed in accordance with the principle of primer design, and were determined to be specific based on Blast comparison (Table 1).

\section{Determination of type II collagen}

Degenerative intervertebral disc nucleus pulposus cells from each group were collected and cell lysate solution was added. After centrifugation at 22,500 $g$ for $5 \mathrm{~min} ; 100 \mu \mathrm{L}$ supernatant was removed and $20 \mu \mathrm{L}$ sodium dodecyl sulfate buffer was added. After boiling 
the samples in a water bath for 10 min to denature the protein, 10 and $8 \%$ sodium dodecyl sulfate-polyacrylamide gel electrophoresis were performed. After separation, the proteins were transferred to a cellulose nitrate membrane, followed by blocking for $30 \mathrm{~min}$. At $37^{\circ} \mathrm{C}$, primary antibody (anti-type II collagen, 1:500) and second antibody (anti-human IgG) were added for hybridization, followed by 3,3'-diaminobenzidine coloration. The digital gel imager ChemiImager 4000 was used to scan the strips, and the gray value of the strips was analyzed using the Quantity one v4.62 software (Bio-Rad, Hercules, CA, USA).

\begin{tabular}{ll}
\multicolumn{2}{c}{ Table 1. Specific primer sequences. } \\
\hline Primer & Sequence \\
\hline F-type II collagen & 5'-ATGACAATCTGGCTCCCAAC-3' \\
R-type II collagen & 5'-ATCCTTCAGGGCAGTGTACG-3' \\
Upstream $\beta$-actin & 5'-CACGATGGAGGGGCCGGACTCATC-3' \\
Downstream $\beta$-actin & 5'-TAAAGACCTCTATGCCAACACAGT-3' \\
\hline
\end{tabular}

\section{RESULTS}

\section{Results of cell culture}

After multi-subculture, adherent growth of nucleus pulposus cells was observed to be in the shape of a polygon. Cell division and proliferation was clear. Because of the low density of the cells, sporadic cells showed a cluster-like distribution. At the 5th generation of subculture, dedifferentiation of cells could be observed and the cell morphology had changed from a round and a polygonal shape to short-shuttle shape, indicating the degeneration stage of cells (Figure 1A, B, C).

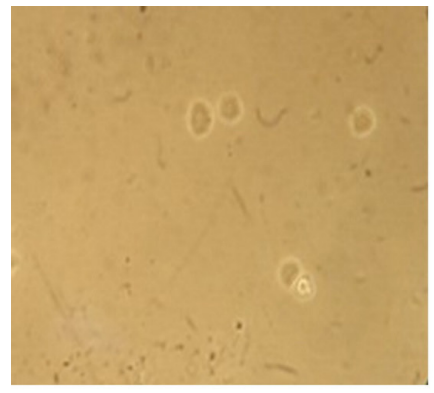

A

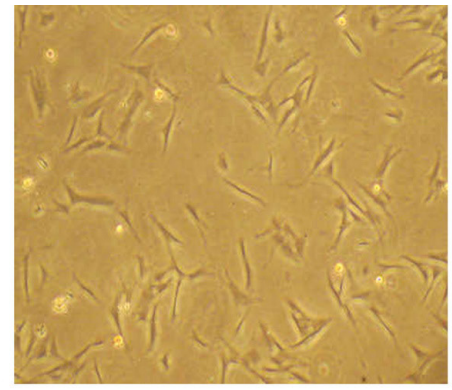

B

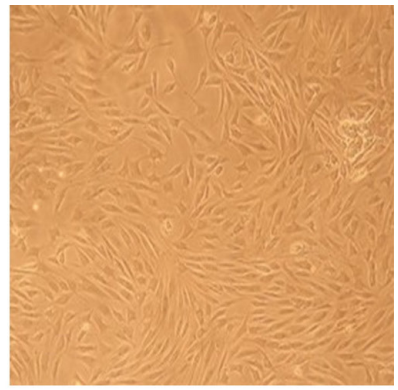

$\mathrm{C}$

Figure 1. Results of cell culture (200X). A. Intervertebral disc nucleus pulposus cells; B. after 3 days of passage; C. cultured to the 5 th generation.

\section{AAV-EGFP transfection efficiency}

After transfection with AAV-EGFP, nucleus pulposus cells showed green fluorescence under a microscope. The transfection efficiency was low, and the positive cells accounted for $15 \%$ of the total cells (Figure 2). 


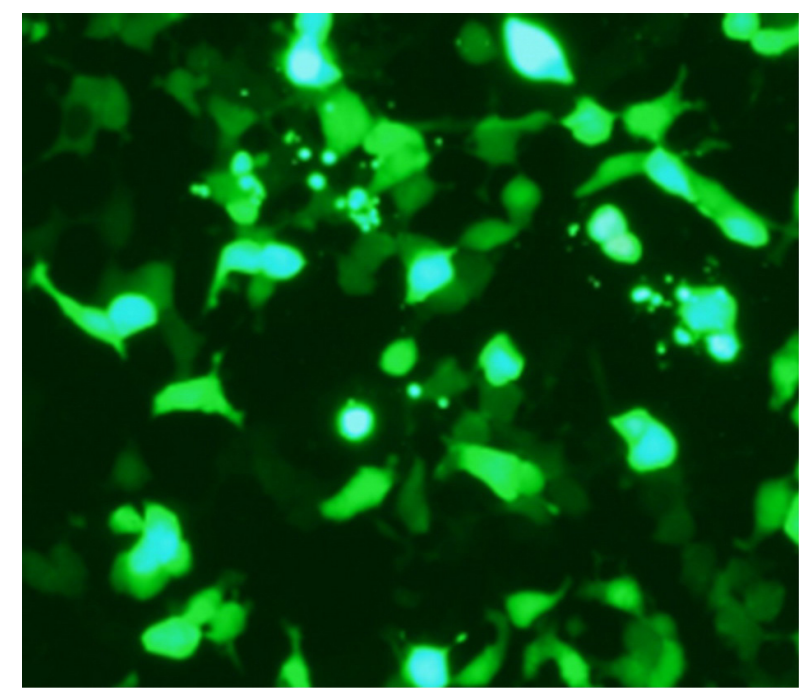

Figure 2. AAV-EGFP tansfection efficiency (200X).

\section{Transfection results}

As shown in Figure 3, after transfection, the SOX9 gene showed red fluorescence, the BMP-7 gene showed green fluorescence, and the double gene showed a mixture of the two colors.

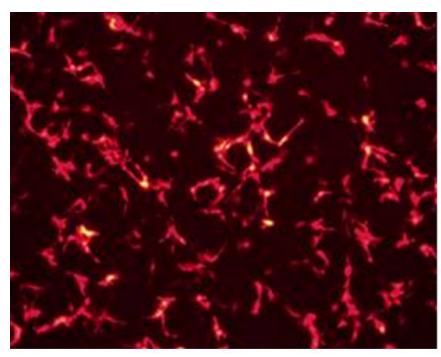

A

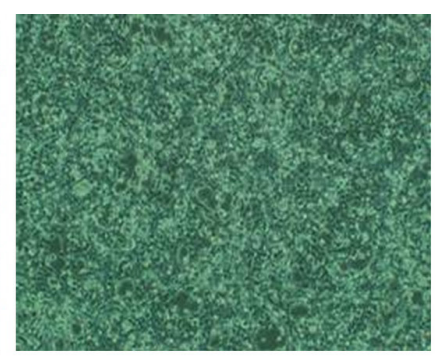

B

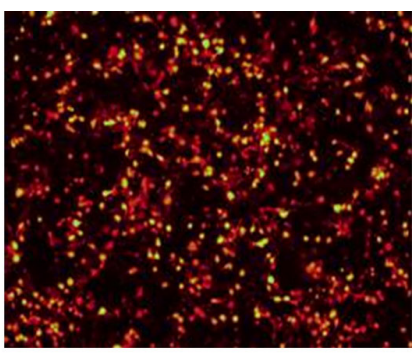

$\mathrm{C}$

Figure 3. Transfection results. A. SOX9 transfection; B. BMP-7 transfection; C. double-gene co-transfection.

\section{Expression of type II collagen mRNA}

After transfection for $48 \mathrm{~h}$, the expression of type II collagen mRNA in the double-gene transfection group was significantly different from that in other groups $(\mathrm{P}<0.05)$. There was no significant difference between the BMP-7 transfection group and SOX9 transfection group. This indicated that BMP-7 and SOX9 double-gene co-transfection up-regulated the expression of type II collagen mRNA in human intervertebral disc nucleus pulposus cells. Double-gene therapy had a synergistic effect on lumbar intervertebral disc degeneration (Figure 4 and Table 2). 
M A $\quad$ B $\quad$ C $\quad$ D

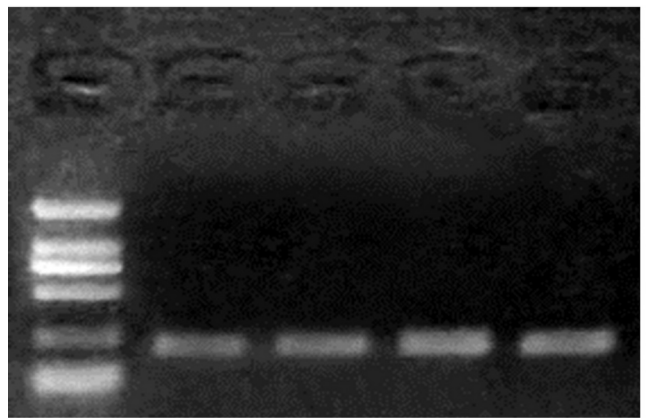

Figure 4. PCR results. Lane $M=$ marker; lane $A=$ blank control; lane $B=\mathrm{BMP}-7$; lane $C=\mathrm{SOX}$; lane $D=$ BMP-7+SOX9.

Table 2. Expression of type II collagen mRNA.

\begin{tabular}{lcccc}
\hline & Blank & BMP-7 & SOX9 & BMP-7+SOX9 \\
\hline$\beta$-actin & 17.30878 & 17.95138 & 18.18228 & 19.39261 \\
$\mathrm{COL} 2 \mathrm{~A} 1$ & 30.19162 & 30.42282 & 30.35106 & 29.97781 \\
$\Delta \mathrm{t}$ & 12.88284 & 12.47144 & 12.16878 & 10.58521 \\
$\Delta \Delta \mathrm{t}$ & 0 & -0.41139 & -2.29763 \\
$2^{-\Delta \Delta \mathrm{t}}$ & 1 & 1.579115 & 2.71405 & 4.328647 \\
\hline$\Delta \mathrm{t}=\mathrm{Ct}_{\text {sample }}-\mathrm{Ct}_{\beta \text {-actin }} ; \Delta \Delta \mathrm{t}_{\text {COL2A1 }}=\Delta \mathrm{t}_{\text {COL2A1 }}-\Delta \mathrm{t}_{\text {blank }}$. & &
\end{tabular}

\section{Expression of type II collagen}

After transfection for $48 \mathrm{~h}$, the expression of type II collagen in the BMP7-transfection group, SOX9 transfection group, and double-gene transfection group were significantly higher than in the blank control group $(\mathrm{P}<0.05)$. The expression level in the double-gene transfection group was significantly higher than in the BMP-7 transfection group and SOX9 transfection group $(\mathrm{P}<0.05)$. This suggested that BMP-7 and SOX9 could promote the synthesis of type II collagen, and this promotion effect with the double gene was more pronounced (Figure 5).

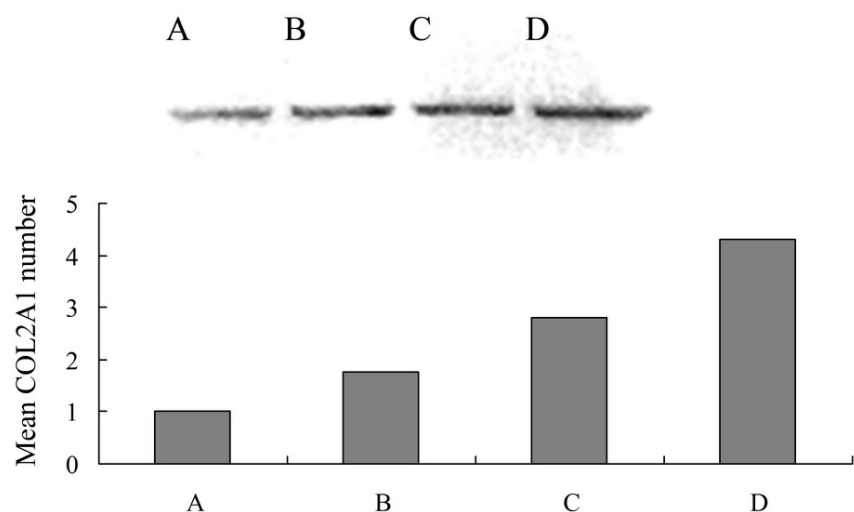

Figure 5. Western blotting results. Columns: $A=$ blank control; $B=B M P-7 ; C=S O X 9 ; D=B M P-7+S O X 9$. 


\section{DISCUSSION}

Intervertebral disc degeneration is a common cause of low back pain. It has serious impact on the physical and mental health of patients and increases the social burden. A series of scientific conservative and surgical strategies are available for the subsequent treatment of diseases resulting from intervertebral disc degeneration. However, these measures cannot slow down or stop intervertebral disc degeneration. With the development of molecular biology techniques, gene therapy has become the dominant method of biological treatment of intervertebral disc degeneration, which promotes the synthesis of major matrix components such as type II collagen and polysaccharide, and decreasing their degradation and for preventing and reverting intervertebral disc degeneration (Xi et al., 2013a). The most common genes targeted for treatment of intervertebral disc degeneration include TGF- $\beta$ (Nishida et al., 1999), SOX9, TIMP (Haro et al., 2000), BMP, and insulin-like growth factor-1 (Osada et al., 1996). Related studies have confirmed that a combination of 2 or more growth factors has a larger effect on the treatment of degenerative intervertebral disc compared with single-gene therapy. Multi-gene co-transfection has a stronger ability for biological control over nucleus pulposus cells in the intervertebral disc (Moon et al., 2008).

BMP-7, also known as osteogenic protein-1 (OP-1), belongs to the TGF superfamily. As an important factor promoting bone fusion, it has been approved by the Food \& Drug Administration and is widely used in clinical practice. Takegami et al. (2002) examined the effect of BMP-7 on the rabbit intervertebral disc. Masuda et al. (2006) found that recombinant human OP-1 can stimulate type II collagen and proteoglycan synthesis as well as increase the expression of proteoglycan and collagen type II mRNA in a dose-dependent manner. Chubinskaya et al. (2007) injected OP-1 into the rat degenerative intervertebral disc; immunohistochemistry studies confirmed that OP-1 increased the extracellular matrix in the degenerative intervertebral disc, altering the cell to have a normal morphology. Shintani and Hunziker (2007) studied the regulatory effect of BMP-7 on chondrogenesis and found that BMP-7 positively regulated the expression of the key chondrogenesis factor SOX9 and type II collagen gene in intervertebral disc cells in a dose-dependent manner.

SOX9 is an important transcription factor in the process of chondrogenesis; it promotes expression of gene products by activating a series of signal transduction pathways. Sive et al. (2002) applied reverse transcription-PCR, Western blotting, and immunological methods to detect SOX9 and type II collagen gene expression in intervertebral disc nucleus pulposus cells at different time points. The expression of SOX9 mRNA in the intervertebral disc nucleus pulposus cells decreased in patients with severe intervertebral disc degeneration. The decreased amplitude of SOX9 was consistent with that observed for type II collagen. This suggests that intervertebral disc degeneration is related to decreased SOX9 expression and that SOX9 plays an important role in the positive regulation of type II collagen synthesis. Paul et al. (2003) found that in adenovirus-mediated SOX9 in vitro transfection of degenerative intervertebral disc cells, SOX9 and type II collagen expression increased. When an adenovirus vector containing SOX9 was injected into a fiber ring acupuncture preparation of a rabbit intervertebral disc degeneration model, the cells in the virus injection group better retained the chondrocyte phenotype than did the control group. Lefebvre et al. (1997) found that a minimum of DNA element in intron I of the type II collagen gene may induce the expression of cartilage-specific cells in transgenic mice. This element may have been a cartilage cell-specific enhancer in the transient transfection experiments. Expression of type II collagen gene is closely related to high expression of SOX9 
RNA and protein in cartilage cells, and this minimum type II collagen enhancer is the target sequence of SOX9. Ren et al. (2013) used SOX9 and OP-1-double gene for in vivo co-transfection of rabbit degenerative intervertebral disc and achieved encouraging results.

In conclusion, therapy using AAV-mediated BMP-7 and SOX9 double gene had a remarkable effect on intervertebral disc degeneration, and even reversed degeneration. This synergistic therapeutic effect is superior to that observed using a single gene. This study has provides a foundation for the further application of double-gene therapy for treating human intervertebral disc degeneration.

\section{ACKNOWLEDGMENTS}

Research supported by the Foundation for Distinguished Young and Middle-Aged Scientists of Shandong Province, China (\#2010BSE01024).

\section{REFERENCES}

Borenstein D (2000). Epidemiology, etiology, diagnostic evaluation, and treatment of low back pain. Curr. Opin. Rheumatol. 4: 226-232.

Chubinskaya S, Kawakami M, Rappoport L, Matsumoto T, et al. (2007). Anti-catabolic effect of OP-1 in chronically compressed intervertebral discs. J. Orthop. Res. 25: 517-530.

Haro H, Crawford HC, Fingleton B, MacDougall JR, et al. (2000). Matrix metalloproteinase-3-dependent generation of a macrophage chemoattractant in model of herniated disc resorption. J. Clin. Incest. 105: 133-141.

Lefebvre V, Huang W, Harley VR, Goodfellow PN, et al. (1997). SOX9 is a potent activator of the chondrocyte-specific enhancer of the proalphal(II) collagen gene. Mol. Cell. Biol. 17: 2336-2346.

Lohr F, Huang Q, Hu K, Dewhirst MW, et al. (2001). Systemic vector leakage and transgene expression by intratumorally injected recombinant adenovirus vectors. Clin. Cancer Res. 7: 3625-3628.

Masuda K, Imai Y, Okuma M, Muehleman C, et al. (2006). Osteogenic protein-1 injection into a degenerated disc induces the restoration of disc height and structural changes in the rabbit anular puncture model. Spine 31: 742-754.

Moon SH, Nishida K, Gilbertson LG, Lee HM, et al. (2008). Biologic response of human intervertebral disc cell to gene therapy cocktail. Spine 33: 1850-1855.

Nishida K, Kang JD, Gilbertson LG, Moon SH, et al. (1999). Modulation of the biologic activity of the rabbit intervertebral disc by gene therapy: an in vivo study of adenovirus-mediated transfer of the human transforming growth factor beta1 encoding gene. Spine 24: 2419-2425.

Osada R, Ohshima H, Ishihara H, Yudoh K, et al. (1996). Autocrine/paracrine mechanism of insulin-like growth factor-1 secretion, and the effect of insulin-like growth factor-1 on proteoglycan synthesis in bovine intervertebral discs. $J$. Orthop. Res. 14: 690-699.

Paul R, Haydon RC, Cheng H, Ishikawa A, et al. (2003). Potential use of S0X9 gene therapy for intervertebral degenerative disc disease. Spine 28: 755-763.

Ren S, Liu YJ, Ma JF, Liu Y, et al. (2013). Treatment of rabbit intervertebral disc degeneration with co-transfection by adeno-associated virus-mediated sox9 and op-1douvle genes in vivo. Int. J. Mol. Med. 32: 1063-1068.

Sai JM, Hu YG and Wang DC (2007). Constructing adeno-associated virus-TGFbeta3 and comparing its biological effect on proteoglycan synthesis in dedifferentiated nucleus pulpous cells with adenovirus-TGFbeta1. Chin. Med. Sci. J. 22: $113-118$.

Shintani N and Hunziker EB (2007). Chondrogenic differentiation of bovine synovium: bone morphogenetic proteins 2 and 7 and transforming growth factor beta1 induce the formation of different types of cartilaginous tissue. Arthritis Rheum. 56: 1869-1879.

Sive JI, Baird P, Jeziorsk M, Watkins A, et al. (2002). Expression of chondrocyte markers by cells of normal and degenerate intervertebral disc. Mol. Pathol. 55: 91-97.

Takegami K, Thonar EJ, An HS, Kamada H, et al. (2002). Osteogenic protein-1 enhances matrix replenishment by intervertebral disc cells previously exposed to interleukin-1. Spine 27: 1318-1325.

Wallach CJ, Gilbertson LG and Kang JD (2003). Gene therapy applications for intervertebral disc degeneration. Spine 28: 93-98. 
Wang JC, Yoo S, Kanim LEA and Mcallister P (1999). Gene therapy for spinal fusion: transformation of bone marrow cells with adenoviral vector to produce BMP-2. Trans. Scoliosis Res. Soc. 34: 50-57.

Wehling P, Schulitz KP, Robbins PD, Evans CH, et al. (1997). Transfer of genes to chondrocytic cells of the lumbar spine. Proposal for a treatment strategy of spinal disorders by local gene therapy. Spine 22: 1092-1097.

Xi Y, Kong J, Liu Y, Wang Z, et al. (2013a). Minimally invasive induction of an early lumbar disc degeneration model in rhesus monkeys. Spine 38: E579-E586.

Xi YM, Dong YF, Wang ZJ, Liu Y, et al. (2013b). Co-transfection of adeno-associated virus-mediated human vascular endothelial growth factor 165 and transforming growth factor- $\beta 1$ into annulus fibrosus cells of rabbit degenerative intervertebral discs. Genet. Mol. Res. 12: 4895-4908. 